\title{
SZEGÖ AND PARA-ORTHOGONAL POLYNOMIALS \\ ON THE REAL LINE: ZEROS AND CANONICAL SPECTRAL TRANSFORMATIONS
}

\author{
KENIER CASTILLO, REGINA LITZ LAMBLÉM, FERNANDO RODRIGO RAFAELI, \\ AND ALAGACONE SRI RANGA
}

\begin{abstract}
We study polynomials which satisfy the same recurrence relation as the Szegö polynomials, however, with the restriction that the (reflection) coefficients in the recurrence are larger than one in modulus. Para-orthogonal polynomials that follow from these Szegö polynomials are also considered. With positive values for the reflection coefficients, zeros of the Szegö polynomials, para-orthogonal polynomials and associated quadrature rules are also studied. Finally, again with positive values for the reflection coefficients, interlacing properties of the Szegö polynomials and polynomials arising from canonical spectral transformations are obtained.
\end{abstract}

\section{INTRODUCTION}

Let $0<a<b<\infty$. We say that the strong positive measure $\psi$, defined on $[a, b]$, belongs to the symmetric class $S^{3}[\xi, \beta, b]$ if

$$
\frac{\mathrm{d} \psi(t)}{t^{\xi}}=-\frac{\mathrm{d} \psi\left(\beta^{2} / t\right)}{\left(\beta^{2} / t\right)^{\xi}}, \quad t \in[a, b],
$$

where $0<\beta<b, a=\beta^{2} / b$ and $2 \xi \in \mathbb{Z}$. The classification of the symmetry (see [2]) is according to the value of $\xi$.

A detailed study of the L-orthogonal polynomials $\left\{\mathcal{Q}_{n}\right\}$ when $\psi$ belongs to the class $S^{3}[0, \beta, b]$ was considered in Sri Ranga, Andrade and McCabe [13. Some other aspects of these polynomials were also treated simultaneously in Common and McCabe 1 .

We now let, without any loss of generality, $\beta=1$ and hence $1 / a=b>1$. We can state the following results when $\psi \in S^{3}[0,1, b]$.

Received by the editor April 20, 2011 and, in revised form, June 12, 2011 and July 19, 2011. 2010 Mathematics Subject Classification. Primary 42C05, 30B70; Secondary 30E05.

Key words and phrases. Szegö polynomials, para-orthogonal polynomials, reflection coefficients, canonical spectral transformations.

This work has been done in the framework of a joint project of Dirección General de Investigación, Ministerio de Educación Ciencia of Spain and the Brazilian Science Foundation CAPES, Project CAPES/DGU 160/08.

The work of the first author was supported by Dirección General de Investigación, Ministerio de Ciencia e Innovación of Spain, grant MTM2009-12740-C03-01.

The work of the second, third and fourth authors was supported by FAPESP under grant $2009 / 13832-9$

The work of the fourth author was supported by CNPq. 
If $f$ is integrable with respect to $\psi$, then

$$
\int_{a}^{b} f(t) \mathrm{d} \psi(t)=\int_{a}^{b} f(1 / t) \mathrm{d} \psi(t) .
$$

The associated L-orthogonal polynomials $\left\{\mathcal{Q}_{n}\right\}$ satisfy the three-term recurrence relation

$$
\mathcal{Q}_{n+1}(z)=\left(z-b_{n+1}\right) \mathcal{Q}_{n}(z)-a_{n+1} z \mathcal{Q}_{n-1}(z), \quad n \geq 1,
$$

with $\mathcal{Q}_{0}(z)=1$ and $\mathcal{Q}_{1}(z)=z-b_{1}$, where the positive coefficients $a_{n}$ and $b_{n}$ satisfy

$$
b_{n} b_{n+1}=\gamma_{n} / \gamma_{n+1}, \quad n \geq 0,
$$

and $\gamma_{n}=b_{n}+a_{n+1}$, with $b_{0}=\gamma_{0}=1$. Consequently,

$$
\mathcal{Q}_{n}(0)=(-1)^{n} b_{1} b_{2} \cdots b_{n}=(-1)^{n} \sqrt{b_{n} / \gamma_{n}}, \quad n \geq 1 .
$$

Moreover, if $\mathcal{S}_{n}(z)=\left[z^{n} \mathcal{Q}_{n}(1 / z)\right] / \mathcal{Q}_{n}(0), \quad n \geq 1$, then

$$
\begin{aligned}
\mathcal{S}_{n}(z) & =\mathcal{Q}_{n}(z)-a_{n+1} \mathcal{Q}_{n-1}(z) \\
& =\left[\mathcal{Q}_{n+1}(z)+b_{n+1} \mathcal{Q}_{n}(z)\right] / z, \quad n \geq 1 .
\end{aligned}
$$
lity)

$$
\int_{a}^{b} t^{-s} \mathcal{S}_{n}(t) \mathrm{d} \psi(t)=0, \quad s=0,1, \ldots, n-1 .
$$

If we define the reciprocal polynomials of $\mathcal{S}_{n}$ by $\mathcal{S}_{n}^{*}(z)=z^{n} \overline{\mathcal{S}_{n}(1 / \bar{z})}$ then, since $S_{n}$ is real, $\mathcal{S}_{n}^{*}(z)=z^{n} \mathcal{S}_{n}(1 / z)=\mathcal{Q}_{n}(z) / \mathcal{Q}_{n}(0), n \geq 1$ and from (1.1),

$$
\mathcal{S}_{n}(z)=z \mathcal{S}_{n-1}(z)+\tilde{\delta}_{n} \mathcal{S}_{n-1}^{*}(z), \quad n \geq 1,
$$

where $\tilde{\delta}_{n}=\left[\mathcal{Q}_{n}(0)\right]^{-1}=(-1)^{n} \sqrt{\gamma_{n} / b_{n}}$ for $n \geq 1$.

Hence, from (1.2) and (1.3), the polynomials $\mathcal{S}_{n}$ can be called the monic Szego" polynomials on the positive interval $[a, b]$. The numbers $\tilde{\delta}_{n}=\mathcal{S}_{n}(0)$, which play the role of reflection coefficients, satisfy

$$
\left|\tilde{\delta}_{n}\right|>1 \text { for } n \geq 1 \text {. }
$$

To be able to talk about Szegö polynomials on a positive interval $[a, b]$ (see also [16]), it is important that the measure is of the class $S^{3}[0,1, b]$, with $a=1 / b$. Otherwise the monic polynomials $\left\{\mathcal{S}_{n}\right\}$ defined on $[a, b]$ by (1.2) do not satisfy (1.3). For example, if the measure $\psi$ is of the class $S^{3}[-1 / 2,1, b]$, then for the monic polynomials $\left\{\mathcal{S}_{n}\right\}$ defined by (1.2) we would have, instead of (1.3),

$$
\mathcal{S}_{n}(z)=\tilde{\delta}_{n} \mathcal{S}_{n}^{*}(z)=z \mathcal{S}_{n-1}(z)+\tilde{\delta}_{n} \mathcal{S}_{n-1}^{*}(z)-\tilde{\alpha}_{n} z \mathcal{S}_{n-2}(z),
$$

for $n \geq 2$, where $\tilde{\delta}_{n}=\mathcal{S}_{n}(0)$ and $\tilde{\alpha}_{n}>0$.

Returning to the class $S^{3}[0,1, b]$, from (1.3)

$$
\mathcal{S}_{n}^{*}(z)=\tilde{\delta}_{n} z \mathcal{S}_{n-1}(z)+\mathcal{S}_{n-1}^{*}(z), \quad n \geq 1 .
$$

One can also obtain from (1.1) that

$$
\mathcal{S}_{n}(z)=\tilde{\delta}_{n} \mathcal{S}_{n}^{*}(z)-\left(\tilde{\delta}_{n}^{2}-1\right) z \mathcal{S}_{n-1}(z), \quad n \geq 1 .
$$


Thus, $(-1)^{n} \mathcal{S}_{n}^{*}(z)$ and $\mathcal{S}_{n}(z), n \geq 0$, are the successive even and odd denominator polynomials of the the special PC-fraction (Peron-Caratháodory continued fraction)

$$
\beta_{0}-\frac{2 \beta_{0}}{1}-\frac{1}{\delta_{1} z}-\frac{\left(\delta_{1}^{2}-1\right) z}{\delta_{1}}-\frac{1}{\delta_{2} z}-\frac{\left(\delta_{2}^{2}-1\right) z}{\delta_{2}}-\frac{1}{\delta_{3} z}-\ldots
$$

where $\delta_{n}=(-1)^{n} \tilde{\delta}_{n}>1, n \geq 1$.

The principal objective of this paper is to consider the properties of the monic polynomials $\mathcal{S}_{n}$ given by the recurrence relation (1.3) under the conditions

$$
\tilde{\delta}_{n} \text { complex and }\left|\tilde{\delta}_{n}\right|>1, \quad n \geq 1 .
$$

For this purpose, an analysis of the above PC-fraction, now with the conditions $\delta_{n}=(-1)^{n} \tilde{\delta}_{n}$ complex and $\left|\delta_{n}\right|>1$, is also considered. The analysis of the PC-fraction shows that these polynomials satisfy the Szegö orthogonality $\mathcal{M}\left[z^{-m} S_{n}(z)\right]=0, \quad 0 \leq m \leq n-1$, where the moment functional $\mathcal{M}$ given by $\mathcal{M}\left[z^{m}\right]=\mu_{m}, m=0, \pm 1, \pm 2, \ldots$, satisfies

$$
\bar{\mu}_{-n}=\mu_{n}, \quad(-1)^{n(n+1) / 2} \Delta_{n}>0, \quad n=0,1,2, \ldots .
$$

Relations between the polynomials $\mathcal{S}_{n}(\omega ; z)=\mathcal{S}_{n}(z)-\omega z \mathcal{S}_{n-1}(z)$ and the paraorthogonal polynomials $\mathcal{S}_{n}(z)+\tau \mathcal{S}_{n}^{*}(z)$ are also treated.

In the two particular cases $\delta_{n}>1, n \geq 1$ and $(-1)^{n} \delta_{n}>1, n \geq 1$, zeros of the Szegö polynomials, para-orthogonal polynomials and the associated quadrature rules have been studied. Finally, again for the two particular cases of values for $\delta_{n}$, interlacing properties of the Szegö polynomials and polynomials arising from canonical spectral transformations are obtained.

\section{Szegö POlYNOMIAls From the PC-FraCtion}

We start with the PC-fraction

$$
\beta_{0}-\frac{2 \beta_{0}}{1}-\frac{1}{\bar{\delta}_{1} z}-\frac{\left(\left|\delta_{1}\right|^{2}-1\right) z}{\delta_{1}}-\frac{1}{\bar{\delta}_{2} z}-\frac{\left(\left|\delta_{2}\right|^{2}-1\right) z}{\delta_{2}}-\frac{1}{\bar{\delta}_{3} z}-\ldots
$$

where we assume that

$$
\beta_{0}>0 \text { and }\left|\delta_{n}\right|>1, \quad n \geq 1 .
$$

The treatment of this PC-fraction considered in this section is similar to that found in [7] where the authors assume that $\left|\delta_{n}\right| \neq 1$. The results given here, in addition to making the paper self-contained, helps the readers to see the specific properties satisfied by the associated Toeplitz and Hankel determinants when $\left|\delta_{n}\right|>1, n \geq 1$.

Let $\left\{A_{n}\right\}$ and $\left\{B_{n}\right\}$ be the sequence of numerator and denominator polynomials of this PC-fraction, respectively. Then these polynomials satisfy the recurrence relations

$$
\begin{aligned}
& \left(\begin{array}{l}
A_{2 n}(z) \\
B_{2 n}(z)
\end{array}\right)=\bar{\delta}_{n} z\left(\begin{array}{l}
A_{2 n-1}(z) \\
B_{2 n-1}(z)
\end{array}\right)-\left(\begin{array}{l}
A_{2 n-2}(z) \\
B_{2 n-2}(z)
\end{array}\right), \\
& \left(\begin{array}{l}
A_{2 n+1}(z) \\
B_{2 n+1}(z)
\end{array}\right)=\delta_{n}\left(\begin{array}{l}
A_{2 n}(z) \\
B_{2 n}(z)
\end{array}\right)-\left(\left|\delta_{n}\right|^{2}-1\right) z\left(\begin{array}{l}
A_{2 n-1}(z) \\
B_{2 n-1}(z)
\end{array}\right),
\end{aligned}
$$


for $n \geq 1$, where $B_{0}(z)=1, B_{1}(z)=1, A_{0}(z)=\beta_{0}$ and $A_{1}(z)=-\beta_{0}$. From these, since $\bar{\delta}_{n} \neq 0$, we get

$$
\left(\begin{array}{l}
A_{2 n+2}(z) \\
B_{2 n+2}(z)
\end{array}\right)=\left(\frac{\bar{\delta}_{n+1}}{\bar{\delta}_{n}} z-1\right)\left(\begin{array}{l}
A_{2 n}(z) \\
B_{2 n}(z)
\end{array}\right)-\frac{\bar{\delta}_{n+1}}{\bar{\delta}_{n}}\left(\left|\delta_{n}\right|^{2}-1\right) z\left(\begin{array}{l}
A_{2 n-2}(z) \\
B_{2 n-2}(z)
\end{array}\right)
$$

for $n \geq 1$, with $B_{0}(z)=1, B_{2}(z)=\bar{\delta}_{1} z-1, A_{0}(z)=\beta_{0}$ and $A_{2}(z)=-\beta_{0}\left(\bar{\delta}_{1} z+1\right)$, and

$$
\left(\begin{array}{l}
A_{2 n+3}(z) \\
B_{2 n+3}(z)
\end{array}\right)=\left(z-\frac{\delta_{n+1}}{\delta_{n}}\right)\left(\begin{array}{l}
A_{2 n+1}(z) \\
B_{2 n+1}(z)
\end{array}\right)-\frac{\delta_{n+1}}{\delta_{n}}\left(\left|\delta_{n}\right|^{2}-1\right) z\left(\begin{array}{l}
A_{2 n-1}(z) \\
B_{2 n-1}(z)
\end{array}\right),
$$

for $n \geq 1$, with $B_{1}(z)=1, B_{3}(z)=z-\delta_{1}, A_{1}(z)=-\beta_{0}$ and $A_{3}(z)=-\beta_{0}\left(z+\delta_{1}\right)$.

From these recurrence relations one can easily observe that both $B_{2 n}$ and $B_{2 n+1}$ are polynomials of exact degree $n$ with non-zero trailing coefficient. Likewise, both $A_{2 n}$ and $A_{2 n+1}$ are polynomials of exact degree $n$ with non-zero trailing coefficient. Precisely,

$$
\begin{array}{ll}
B_{2 n}(z)=\bar{\delta}_{n} z^{n}+\ldots+(-1)^{n}, & B_{2 n+1}(z)=z^{n}+\ldots+(-1)^{n} \delta_{n}, \\
A_{2 n}(z)=-\beta_{0}\left[\bar{\delta}_{n} z^{n}+\ldots+(-1)^{n-1}\right], & A_{2 n+1}(z)=-\beta_{0}\left[z^{n}+\ldots+(-1)^{n-1} \delta_{n}\right],
\end{array}
$$

for $n \geq 1$. Using the above recurrence relations one can also easily conclude that

$$
\begin{aligned}
& A_{2 n+1}^{*}(z)=z^{n} \overline{A_{2 n+1}(1 / \bar{z})}=z^{n} \bar{A}_{2 n+1}(1 / z)=(-1)^{n+1} A_{2 n}(z), \\
& B_{2 n+1}^{*}(z)=z^{n} \overline{B_{2 n+1}(1 / \bar{z})}=z^{n} \bar{B}_{2 n+1}(1 / z)=(-1)^{n} B_{2 n}(z),
\end{aligned}
$$

Also from these recurrence relations, the following hold:

$$
\begin{aligned}
& \frac{A_{2 n+2}(z)}{B_{2 n+2}(z)}-\frac{A_{2 n}(z)}{B_{2 n}(z)}=\left\{\begin{array}{l}
\left(2 \beta_{0} \bar{\delta}_{n+1} \prod_{r=1}^{n}\left(\left|\delta_{r}\right|^{2}-1\right)\right) z^{n+1}+O\left(z^{n+2}\right) \\
-\left(2 \beta_{0} \bar{\delta}_{n}^{-1} \prod_{r=1}^{n}\left(\left|\delta_{r}\right|^{2}-1\right)\right) z^{-n}+O\left(z^{-(n+1)}\right),
\end{array}\right. \\
& \frac{A_{2 n+3}(z)}{B_{2 n+3}(z)}-\frac{A_{2 n+1}(z)}{B_{2 n+1}(z)}=\left\{\begin{array}{l}
\left(2 \beta_{0} \delta_{n}^{-1} \prod_{r=1}^{n}\left(\left|\delta_{r}\right|^{2}-1\right)\right) z^{n}+O\left(z^{n+1}\right) \\
-\left(2 \beta_{0} \delta_{n+1} \prod_{r=1}^{n}\left(\left|\delta_{r}\right|^{2}-1\right)\right) z^{-(n+1)}+O\left(z^{-(n+2)}\right),
\end{array}\right. \\
& \frac{A_{2 n+1}(z)}{B_{2 n+1}(z)}-\frac{A_{2 n}(z)}{B_{2 n}(z)}=\left\{\begin{array}{l}
-\left(2 \beta_{0} \delta_{n}^{-1} \prod_{r=1}^{n}\left(\left|\delta_{r}\right|^{2}-1\right)\right) z^{n}+O\left(z^{n+1}\right) \\
-\left(2 \beta_{0} \bar{\delta}_{n}^{-1} \prod_{r=1}^{n}\left(\left|\delta_{r}\right|^{2}-1\right)\right) z^{-n}+O\left(z^{-(n+1)}\right),
\end{array}\right.
\end{aligned}
$$

for $n \geq 1$. Thus we can conclude that there exist a pair of formal power series

$$
L_{0}(z)=\mu_{0}+\sum_{s=1}^{\infty} 2 \mu_{-s} z^{s} \quad \text { and } \quad L_{\infty}(z)=-\mu_{0}-\sum_{s=1}^{\infty} 2 \mu_{s} z^{-s},
$$


with $\mu_{0}=\beta_{0}$ and $\bar{\mu}_{-1}=\mu_{1}=\beta_{0} \delta_{1}$, such that

$$
L_{0}(z)-\frac{A_{2 n}(z)}{B_{2 n}(z)}=\left(2 \beta_{0} \bar{\delta}_{n+1} \prod_{r=1}^{n}\left(\left|\delta_{r}\right|^{2}-1\right)\right) z^{n+1}+O\left(z^{n+2}\right),
$$

$$
\left.L_{\infty}(z)-\frac{A_{2 n}(z)}{B_{2 n}(z)}\right]=-\left(2 \beta_{0} \bar{\delta}_{n}^{-1} \prod_{r=1}^{n}\left(\left|\delta_{r}\right|^{2}-1\right)\right) z^{-n}+O\left(z^{-(n+1)}\right)
$$

$$
\begin{aligned}
L_{0}(z)-\frac{A_{2 n+1}(z)}{B_{2 n+1}(z)}=\left(2 \beta_{0} \delta_{n}^{-1} \prod_{r=1}^{n}\left(\left|\delta_{r}\right|^{2}-1\right)\right) z^{n}+O\left(z^{n+1}\right), \\
L_{\infty}(z)-\frac{A_{2 n+1}(z)}{B_{2 n+1}(z)}=-\left(2 \beta_{0} \delta_{n+1} \prod_{r=1}^{n}\left(\left|\delta_{r}\right|^{2}-1\right)\right) z^{-(n+1)}+O\left(z^{-(n+2)}\right),
\end{aligned}
$$

for $n \geq 1$. That is, the PC-fraction (2.1) corresponds to the formal power series expansions $L_{0}$ and $L_{\infty}$. Now using the reciprocal property (2.3), we also conclude that these formal power series expansions are such that

$$
\bar{\mu}_{-s}=\mu_{s}, \quad s=1,2,3, \ldots .
$$

If we write $B_{2 n+1}(z)=\sum_{r=0}^{n} b_{n, r} z^{r}$ and $A_{2 n+1}(z)=\sum_{r=0}^{n} a_{n, r} z^{r}$, then from (2.5),

$$
\begin{aligned}
L_{0}(z) \sum_{r=0}^{n} b_{n, r} z^{r}-\sum_{r=0}^{n} a_{n, r} z^{r} & =\gamma_{n} z^{n}+O\left(z^{n+1}\right), \\
L_{\infty}(z) \sum_{r=0}^{n} b_{n, r} z^{r-n}-\sum_{r=0}^{n} a_{n, r} z^{r-n} & =O\left(z^{-n-2}\right),
\end{aligned}
$$

for $n \geq 1$, where $\gamma_{n}=(-1)^{n} 2 \beta_{0} \prod_{r=1}^{n}\left(\left|\delta_{r}\right|^{2}-1\right)$. This leads to the linear system

$$
\left[\begin{array}{ccccc}
\mu_{0} & \mu_{1} & \cdots & \mu_{n-1} & \mu_{n} \\
\mu_{-1} & \mu_{0} & \cdots & \mu_{n-2} & \mu_{n-1} \\
\vdots & \vdots & & \vdots & \vdots \\
\mu_{-n+1} & \mu_{-n+2} & \cdots & \mu_{0} & \mu_{1} \\
\mu_{-n} & \mu_{-n+1} & \cdots & \mu_{-1} & \mu_{0}
\end{array}\right]\left[\begin{array}{c}
b_{n, 0} \\
b_{n, 1} \\
\vdots \\
b_{n, n-1} \\
b_{n, n}
\end{array}\right]=\frac{1}{2}\left[\begin{array}{c}
0 \\
0 \\
\vdots \\
0 \\
\gamma_{n}
\end{array}\right]
$$

Applying Cramer's rule for the coefficient $b_{n, n}(=1)$ yields

$$
\Delta_{n}=\left[(-1)^{n} \beta_{0} \prod_{r=1}^{n}\left(\left|\delta_{r}\right|^{2}-1\right)\right] \Delta_{n-1}, \quad n \geq 1,
$$

where $\Delta_{0}=\mu_{0}=\beta_{0}$ and

$$
\Delta_{n}=\operatorname{det}\left[\begin{array}{ccccc}
\mu_{0} & \mu_{1} & \cdots & \mu_{n-1} & \mu_{n} \\
\mu_{-1} & \mu_{0} & \cdots & \mu_{n-2} & \mu_{n-1} \\
\vdots & \vdots & & \vdots & \\
\mu_{-n+1} & \mu_{-n+2} & \cdots & \mu_{0} & \mu_{1} \\
\mu_{-n} & \mu_{-n+1} & \cdots & \mu_{-1} & \mu_{0}
\end{array}\right], \quad n \geq 1 .
$$


Hence, if $\beta_{0}>0$, then $\Delta_{0}=\beta_{0}>0$ and

$$
(-1)^{n(n+1) / 2} \Delta_{n}=\beta_{0}^{n+1} \prod_{m=1}^{n}\left[\prod_{r=1}^{m}\left(\left|\delta_{r}\right|^{2}-1\right)\right]>0, \quad n \geq 1 .
$$

Since $(-1)^{n(n+1) / 2}=(-1)^{\lfloor(n+1) / 2\rfloor}$, if we look at the Hankel determinants

$$
H_{1}^{(0)}=\mu_{0}, \quad H_{n+1}^{(-n)}=\operatorname{det}\left[\begin{array}{cccc}
\mu_{-n} & \mu_{-n+1} & \cdots & \mu_{0} \\
\mu_{-n+1} & \mu_{-n+2} & \cdots & \mu_{1} \\
\vdots & \vdots & & \vdots \\
\mu_{0} & \mu_{1} & \cdots & \mu_{n}
\end{array}\right]
$$

that can be obtain by a rearrangement of rows of the Toeplitz determinants $\Delta_{n}$, we have

$$
H_{n+1}^{(-n)}>0, \quad n \geq 0
$$

Applying Cramer's rule in (2.9) for the coefficient $b_{n, 0}=(-1)^{n} \delta_{n}$, we obtain

$$
\delta_{n}=\frac{1}{\Delta_{n-1}} \operatorname{det}\left[\begin{array}{ccc}
\mu_{1} & \cdots & \mu_{n} \\
\mu_{0} & \cdots & \mu_{n-1} \\
\vdots & & \vdots \\
\mu_{-n+2} & \cdots & \mu_{1}
\end{array}\right]=\frac{1}{\Delta_{n-1}} \operatorname{det}\left[\begin{array}{ccc}
\mu_{-1} & \cdots & \mu_{n-2} \\
\mu_{-2} & \cdots & \mu_{n-3} \\
\vdots & & \vdots \\
\mu_{-n} & \cdots & \mu_{-1}
\end{array}\right] .
$$

The rightmost determinant expression follows by considering the transpose and then using $\bar{\mu}_{-s}=\mu_{s}$. Hence, through an interchanging of rows in the determinants,

$$
H_{n}^{(-n+2)}=\overline{H_{n}^{(-n)}}=\delta_{n} H_{n}^{(-n+1)}, \quad n \geq 1 .
$$

Since $\left|\delta_{n}\right|>1$, this means

$$
\left|H_{n}^{(-n+2)}\right|=\left|H_{n}^{(-n)}\right|>H_{n}^{(-n+1)}>0, \quad n \geq 1 .
$$

Now with respect to the formal power series expansions $L_{0}$ and $L_{\infty}$ in (2.4), we define the linear functional $\mathcal{M}$ on the space of Laurent polynomials by

If $l_{p, q}(z)=\sum_{m=p}^{q} c_{m} z^{m}, c_{m} \in \mathcal{C}$ and $-\infty<p \leq q<\infty$, then $\mathcal{M}\left[l_{p, q}\right]=\sum_{m=p}^{q} c_{m} \mu_{m}$.

Then from (2.9), we have for the monic polynomials $S_{n}=B_{2 n+1}, n=0,1,2, \ldots$,

$$
\mathcal{M}\left[t^{-m} S_{n}\right]=\frac{1}{2} \gamma_{n} \delta_{n, m}, \quad 0 \leq m \leq n, \quad n=1,2,3, \ldots .
$$

Here, $\delta_{n, m}$ represents the Kronecker delta. Also from (2.2) and (2.3),

$$
S_{n}(z)=z S_{n-1}(z)+(-1)^{n} \delta_{n} S_{n-1}^{*}(z), \quad n \geq 1,
$$

with $S_{0}(z)=1, S_{1}(z)=z-\delta_{1}$. Based on these, we refer to $\left\{S_{n}=B_{2 n+1}\right\}$ as the sequence of Szegö polynomials generated by the PC-fraction (2.1). 


\section{SZEGŐ POLYNOMIALS FROM SERIES EXPANSIONS}

Let

$$
L_{0}(z)=\mu_{0}+\sum_{s=1}^{\infty} 2 \mu_{-s} z^{s} \quad \text { and } \quad L_{\infty}(z)=-\mu_{0}-\sum_{s=1}^{\infty} 2 \mu_{s} z^{-s},
$$

be given formal power series expansions such that

$$
\bar{\mu}_{-n}=\mu_{n}, \quad n=1,2,3, \ldots
$$

and the associated Toeplitz determinants, as defined by (2.10), with the property

$$
(-1)^{n(n+1) / 2} \Delta_{n}>0, \quad n=0,1,2, \ldots .
$$

In terms of the Hankel determinants, this last property can be given as

$$
H_{n+1}^{(-n)}>0, \quad n=0,1,2, \ldots .
$$

Now we consider the linear functional $\mathcal{M}$ as defined in the last section. The linear functional $\mathcal{M}$ is called a moment functional and the numbers, $\mu_{s}, s=0, \pm 1, \pm 2, \ldots$ are called the moments associated with this moment functional. Under the conditions (3.1) and (3.2), $\mathcal{M}$ will be referred to as an sq-definite moment functional, meaning "special quasi-definite moment functional". When the moments are also real, then the name rsq-definite moment functional will be used.

The sequence of polynomials $\left\{S_{n}\right\}$ will be defined by $S_{0}=1$ and $S_{n}$ a monic polynomial of degree $n$ such that

$$
\mathcal{M}\left[t^{-m} S_{n}\right]=0, \quad 0 \leq m \leq n-1, \quad n \geq 1 .
$$

Then under the conditions of (3.2) these polynomials always exist. In fact, the following results can be easily established:

$$
\begin{gathered}
S_{n}(z)=\frac{1}{\Delta_{n-1}}\left|\begin{array}{ccccc}
\mu_{0} & \mu_{1} & \cdots & \mu_{n-1} & \mu_{n} \\
\mu_{-1} & \mu_{0} & \cdots & \mu_{n-2} & \mu_{n-1} \\
\vdots & \vdots & & \vdots & \\
\mu_{-n+1} & \mu_{-n+2} & \cdots & \mu_{0} & \mu_{1} \\
1 & z & \cdots & z^{n-1} & z^{n}
\end{array}\right|, \quad n \geq 1, \\
\mathcal{M}\left[t^{-m} S_{n}\right]=\mathcal{M}\left[t^{-n+m} S_{n}^{*}\right]=\delta_{n, m} \frac{\Delta_{n}}{\Delta_{n-1}}, \quad 0 \leq m \leq n, \quad n \geq 1 .
\end{gathered}
$$

The information on $\mathcal{M}\left[t^{-n+m} S_{n}^{*}\right]$ follows from (3.1), as with this symmetric property we have

$$
\overline{\mathcal{M}\left[t^{-m} l_{p, q}(t)\right]}=\mathcal{M}\left[t^{m} \overline{l_{p, q}}(1 / t)\right],
$$

for any Laurent polyomial $l_{p, q}$.

We call $S_{n}$ the Szegö polynomials associated with the sq-definite moment functional $\mathcal{M}$.

Theorem 3.1. Let $\delta_{n}=(-1)^{n} S_{n}(0)$ for $n \geq 1$, where $S_{n}$ are the Szegö polynomials associated with the sq-definite moment functional $\mathcal{M}$. Then the following hold:

(1) The polynomials $S_{n}$ satisfy the recurrence relations

$$
\begin{aligned}
& S_{n}^{*}(z)=(-1)^{n} \bar{\delta}_{n} z S_{n-1}(z)+S_{n-1}^{*}(z), \\
& S_{n}(z)=(-1)^{n} \delta_{n} S_{n}^{*}(z)-\left(\left|\delta_{n}\right|^{2}-1\right) z S_{n-1}(z), \quad n \geq 1 .
\end{aligned}
$$

(2) $\left|\delta_{n}\right|>1$ for all $n \geq 1$. 
(3) If we set $B_{2 n}(z)=(-1)^{n} S_{n}^{*}(z), \quad B_{2 n+1}(z)=S_{n}(z), \quad n=0,1,2, \ldots$, then $\left\{B_{n}\right\}$ are the sequence of denominator polynomials of the PC-fraction (2.1), with $\delta_{n}=(-1)^{n} S_{n}(0)$.

Proof. The proof of this theorem is similar to analogous results in [7]. However, to be able to clarify part (2) of the theorem, we give here the whole proof. For $n \geq 1$ set

where

$$
A(z)=S_{n}^{*}(z)-\alpha_{n} z S_{n-1}(z)-S_{n-1}^{*}(z)
$$

$$
\alpha_{n}=-\frac{\mathcal{M}\left[t^{-n} S_{n-1}^{*}\right]}{\mathcal{M}\left[t^{-(n-1)} S_{n-1}\right]} .
$$

We consider $A$ as a polynomial of degree $n$ and show that it is identically zero. Clearly,

$$
\mathcal{M}\left[t^{-m} A\right]=0 \text { for } 1 \leq m \leq n
$$

Since $A(0)=0$, we can write $A^{*}(z)=\sum_{k=0}^{n-1} a_{k} S_{k}(z)$ and hence

$$
A(z)=\sum_{k=0}^{n-1} \bar{a}_{k} z^{n-k} S_{k}^{*}(z)
$$

Using here the results of (3.6) for $m=n, m=n-1$ until $m=1$ and noting at each stage that $\mathcal{M}\left[S_{n-m}^{*}\right] \neq 0$, we successively obtain that $a_{0}=0, a_{1}=0$ until $a_{n-1}=0$. Hence proving $A(z) \equiv 0$ and thus

$$
S_{n}^{*}(z)=\alpha_{n} z S_{n-1}(z)+S_{n-1}^{*}(z) .
$$

Comparing the coefficients of $z^{n}$ we obtain $\bar{\alpha}_{n}=S_{n}(0)$, thus establishing the first of the recurrence relations.

Now to prove the second recurrence relation, for $n \geq 1$ we set

$$
A(z)=S_{n}(z)-(-1)^{n} \delta_{n} S_{n}^{*}(z)+\alpha_{n} z S_{n-1}(z),
$$

with $\alpha_{n}=-\mathcal{M}\left[t^{-n} S_{n}\right] / \mathcal{M}\left[t^{-(n-1)} S_{n-1}\right]$. Hence,

$$
\mathcal{M}\left[t^{-m} A\right]=0 \text { for } 1 \leq m \leq n .
$$

Since $A(0)=0$, we can follow the exact procedure as before and establish that $A(z) \equiv 0$ to obtain

$$
S_{n}(z)=(-1)^{n} \delta_{n} S_{n}^{*}(z)-\alpha_{n} z S_{n-1}(z) .
$$

Comparing the coefficients of $z^{n}$ we obtain $\bar{\alpha}_{n}=\left|\delta_{n}\right|^{2}-1$, thus establishing the second of the recurrence relations.

Now since $\mathcal{M}\left[t^{-n} S_{n}\right]=\Delta_{n} / \Delta_{n-1}$, we obtain from the above recurrence relation

$$
\left|\delta_{n}\right|^{2}-1=-\frac{\Delta_{n} \Delta_{n-2}}{\Delta_{n-1}^{2}}, \quad n \geq 1 .
$$

Here, $\Delta_{-1}$ is taken to be unity. We can easily verify that (3.2) is sufficient to establish that $\left|\delta_{n}\right|^{2}-1>0, n \geq 1$, and with this part, (2) of the theorem.

To verify part (3), we note that by setting $B_{2 n}(z)=(-1)^{n} S_{n}^{*}(z), B_{2 n+1}(z)=$ $S_{n}(z), n=0,1,2, \ldots$, we obtain from part (1) of this theorem,

$$
\begin{aligned}
& B_{2 n}(z)=\bar{\delta}_{n} z B_{2 n-1}(z)-B_{2 n-2}(z), \\
& B_{2 n+1}(z)=\delta_{n} B_{2 n}(z)-\left(\left|\delta_{n}\right|^{2}-1\right) z B_{2 n-1}(z),
\end{aligned} \quad n \geq 1,
$$


with $B_{0}(z)=B_{1}(z)=1$. Hence, from the theory of continued fractions the result follows.

Since $\delta_{n}=(-1)^{n} S_{n}(0)$, from (3.3) we obtain, as in (2.11),

$$
\delta_{n}=\frac{H_{n}^{(-n+2)}}{H_{n}^{(-n+1)}}=\frac{\overline{H_{n}^{(-n)}}}{H_{n}^{(-n+1)}}, \quad n \geq 1 .
$$

Since $\delta_{n} \neq 0$, from part $(1)$ of the above theorem, the following recurrence relation always holds:

$$
S_{n+1}(z)=\left(z-\beta_{n+1}\right) S_{n}(z)-\alpha_{n+1} z S_{n-1}(z), \quad n \geq 1,
$$

with $S_{0}=1$ and $S_{1}(z)=z-\beta_{1}$, where $\beta_{1}=\delta_{1}$ and for $n \geq 1, \alpha_{n+1}=\beta_{n+1}\left(\left|\delta_{n}\right|^{2}-1\right)$ and $\beta_{n+1}=\frac{\delta_{n+1}}{\delta_{n}}$.

Now we define the polynomials $R_{n}, n \geq 0$, by

$$
R_{0}=-\mu_{0}, \quad R_{n}(z)=\mathcal{M}\left[\frac{z+t}{z-t}\left(S_{n}(t)-S_{n}(z)\right)\right], \quad n \geq 1 .
$$

It is easily verified that $R_{n}$ is a polynomial of exact degree $n$. Precisely,

$$
R_{n}(z)=-\mu_{0}\left[z^{n}+\ldots+(-1)^{n-1} \delta_{n}\right] .
$$

Theorem 3.2. Let $S_{n}$ be the Szego" polynomials associated with the sq-definite moment functional $\mathcal{M}$. Then the polynomials $R_{n}$ defined by (3.9) satisfy the following:

(1) $\quad R_{n}(z)=\mathcal{M}\left[\frac{z+t}{z-t}\left(\frac{z^{k}}{t^{k}} S_{n}(t)-S_{n}(z)\right)\right], \quad 0 \leq k \leq n-1, \quad n \geq 1$.

(2) $\quad-R_{n}^{*}(z)=\mathcal{M}\left[\frac{z+t}{z-t}\left(\frac{z^{k}}{t^{k}} S_{n}^{*}(t)-S_{n}^{*}(z)\right)\right], \quad 1 \leq k \leq n, \quad n \geq 1$.

(3) The following recurrence relations hold:

$$
\begin{aligned}
& {\left[-R_{n}^{*}(z)\right]=(-1)^{n} \bar{\delta}_{n} z R_{n-1}(z)+\left[-R_{n-1}^{*}(z)\right], \quad n \geq 1 .} \\
& R_{n}(z)=(-1)^{n} \delta_{n}\left[-R_{n}^{*}(z)\right]-\left(\left|\delta_{n}\right|^{2}-1\right) z R_{n-1}(z),
\end{aligned}
$$

(4) For $n \geq 0$, if we set $A_{2 n}(z)=(-1)^{n+1} R_{n}^{*}(z), A_{2 n+1}(z)=R_{n}(z)$, then $\left\{A_{n}\right\}$ are the sequence of numerator polynomials of the PC-fraction (2.1), with $\beta_{0}=\mu_{0}$ and $\delta_{n}=(-1)^{n} S_{n}(0), n \geq 1$.

Proof. The proof of the results in this theorem is the same as similar results found in 7 .

From the above theorem it also follows that the polynomials $R_{n}$ satisfy the three term recurrence relation

$$
R_{n+1}(z)=\left(z-\beta_{n+1}\right) R_{n}(z)-\alpha_{n+1} z R_{n-1}(z), \quad n \geq 1
$$

with $R_{0}=-\mu_{0}$ and $R_{1}(z)=-\mu_{0}\left(z+\delta_{1}\right)$. The values of $\alpha_{n}$ and $\beta_{n}$ are as in (3.8). 


\section{Para-orthogonal polynomials}

Given the parameter $\omega \in \mathbb{C}$, we consider the sequence of polynomials $\left\{S_{n}(\omega ;).\right\}$ and $\left\{R_{n}(\omega ;).\right\}$ given by $S_{0}(\omega ; z)=1, R_{0}(\omega ; z)=-\mu_{0}$ and

$$
S_{n}(\omega ; z)=S_{n}(z)-\omega z S_{n-1}(z), \quad R_{n}(\omega ; z)=R_{n}(z)-\omega z R_{n-1}(z), \quad n \geq 1 .
$$

Clearly, $S_{n}(0 ; z)=S_{n}(z)$ and for any $\omega$,

$$
S_{n}(\omega ; 0)=S_{n}(0)=(-1)^{n} \delta_{n} \neq 0, \quad n \geq 1 .
$$

Theorem 4.1. Let $\delta_{n}=(-1)^{n} S_{n}(0)$ for $n \geq 1$, where $S_{n}$ are the Szegö polynomials associated with the sq-definite moment functional $\mathcal{M}$. Then the following hold:

$$
\begin{aligned}
& \text { (1) } \mathcal{M}\left[t^{-m} S_{n}(\omega ; t)\right]=0, \quad 1 \leq m \leq n-1 . \\
& \text { (2) } R_{n}(\omega ; z)=\mathcal{M}\left[\frac{z+t}{z-t}\left(\frac{z^{k}}{t^{k}} S_{n}(\omega ; t)-S_{n}(\omega ; z)\right)\right], \quad 1 \leq k \leq n-1, \quad n \geq 2 . \\
& \text { (3) }-R_{n}^{*}(\omega ; z)=\mathcal{M}\left[\frac{z+t}{z-t}\left(\frac{z^{k}}{t^{k}} S_{n}^{*}(\omega ; t)-S_{n}^{*}(\omega ; z)\right)\right], \quad 1 \leq k \leq n-1, \quad n \geq 2 .
\end{aligned}
$$

Proof. The proof of part (1) is immediate. To prove part (2), we can write, using part (1) of Theorem 3.2 .

$$
\begin{aligned}
& R_{n}(\omega ; z)= R_{n}(z)-\omega z R_{n-1}(z) \\
&=\mathcal{M}\left[\frac{z+t}{z-t}\left(\frac{z^{k}}{t^{k}} S_{n}(t)-S_{n}(z)\right)\right] \\
& \quad-\omega z \mathcal{M}\left[\frac{z+t}{z-t}\left(\frac{z^{k-1}}{t^{k-1}} S_{n-1}(t)-S_{n-1}(z)\right)\right],
\end{aligned}
$$

$1 \leq k \leq n-1, n \geq 2$. This leads us to the required result.

Similarly, part (3) of this theorem follows from part (2) of Theorem 3.2

For convenience, we write $\widehat{S}_{n}(\omega ; z)=\bar{S}_{n}(z)-\bar{\omega} z \bar{S}_{n-1}(z)$ and $\widehat{R}_{n}(\omega ; z)=\bar{R}_{n}(z)-$ $\bar{\omega} z \bar{R}_{n-1}(z), n \geq 1$. Hence, observe that

$$
S_{n}^{*}(\omega ; z)=z^{n} \overline{S_{n}(\omega ; 1 / \bar{z})}=z^{n} \widehat{S}_{n}(\omega ; 1 / z)=S_{n}^{*}(z)-\bar{\omega} S_{n-1}^{*}(z), \quad n \geq 1 .
$$

From the recurrence relations for $S_{n}$ in Theorem 3.1, we get

$$
S_{n}(1 ; z)=(-1)^{n} \delta_{n} S_{n-1}^{*}(0 ; z), \quad S_{n}\left(-\left|\delta_{n}\right|^{2}+1 ; z\right)=(-1)^{n} \delta_{n} S_{n}^{*}(0 ; z),
$$

for $n \geq 1$.

Theorem 4.2. Let $\delta_{n}=(-1)^{n} S_{n}(0)$ for $n \geq 1$, where $S_{n}$ are the Szegö polynomials associated with the sq-definite moment functional $\mathcal{M}$. If $\bar{\nu}=\frac{1-\omega-\left|\delta_{n}\right|^{2}}{1-\omega}$, then

$$
S_{n}^{*}(\nu ; z)=z^{n} \widehat{S}_{n}(\nu ; 1 / z)=\frac{1-\bar{\nu}}{(-1)^{n} \delta_{n}} S_{n}(\omega ; z), \quad n \geq 1
$$

$$
\begin{aligned}
& R_{n}^{*}(\nu ; z)=z^{n} \widehat{R}_{n}(\nu ; 1 / z)=\frac{1-\bar{\nu}}{(-1)^{n-1} \delta_{n}} R_{n}(\omega ; z), \quad n \geq 1, \\
& \frac{d S_{n}^{*}(\nu ; z)}{d z}=n z^{n-1} \widehat{S}_{n}(\nu ; 1 / z)-z^{n-2} \widehat{S}_{n}^{\prime}(\nu ; 1 / z)=\frac{1-\bar{\nu}}{(-1)^{n} \delta_{n}} S_{n}^{\prime}(\omega ; z), \quad n \geq 1,
\end{aligned}
$$


In particular, if $\omega_{n}=1-\sigma \bar{\delta}_{n}$, with $|\sigma|=1$, then

$$
S_{n}^{*}\left(\omega_{n} ; z\right)=z^{n} \widehat{S}_{n}\left(\omega_{n} ; 1 / z\right)=(-1)^{n} \bar{\sigma} S_{n}\left(\omega_{n} ; z\right), \quad n \geq 1,
$$

$$
R_{n}^{*}\left(\omega_{n} ; z\right)=z^{n} \widehat{R}_{n}\left(\omega_{n} ; 1 / z\right)=(-1)^{n-1} \bar{\sigma} R_{n}\left(\omega_{n} ; z\right), \quad n \geq 1
$$

(6) $\quad n z^{n-1} \widehat{S}_{n}\left(\omega_{n} ; 1 / z\right)-z^{n-2} \widehat{S}_{n}^{\prime}\left(\omega_{n} ; 1 / z\right)=(-1)^{n} \bar{\sigma} S_{n}^{\prime}\left(\omega_{n} ; z\right), \quad n \geq 1$,

Here $\widehat{S}_{n}^{\prime}(\omega ; z)=\frac{d \widehat{S}_{n}(\omega ; z)}{d z}$ and $S_{n}^{\prime}(\omega ; z)=\frac{d S_{n}(\omega ; z)}{d z}$.

Proof. Since $\omega$ and $\nu$ are different from 1, the proof of part (1) follows from the relations in (4.1). Part (2), especially when $n \geq 2$, follows from Theorem 4.1. Part (3) is obtained from part (1) of this theorem by straightforward differentiation. To obtain the remaining parts, we let $\omega=\nu=\omega_{n}$, and this gives $\left|\omega_{n}-1\right|^{2}=\left|\delta_{n}\right|^{2}$.

Note that we can also write,

$$
S_{n}\left(\omega_{n} ; z\right)=S_{n}\left(1-\sigma \bar{\delta}_{n} ; z\right)=\frac{\left|\delta_{n}\right|^{2}-\sigma \bar{\delta}_{n}}{\left|\delta_{n}\right|^{2}-1}\left[S_{n}(z)+\tau S_{n}^{*}(z)\right]
$$

where $\tau=(-1)^{n} \frac{\delta_{n}}{\bar{\delta}_{n}} \frac{1-\sigma \bar{\delta}_{n}}{\sigma-\delta_{n}}$.

Again, following the nomenclature used in the case of the classical Szegö polynomials (see [7]), we will refer to the polynomials $S_{n}+\tau S_{n}^{*}$, when $|\tau|=1$, as the para-orthogonal polynomials.

Since $|\tau|=1$ whenever $|\sigma|=1$, the polynomials $S_{n}\left(\omega_{n} ;.\right)$ are hence paraorthogonal polynomials multiplied by constant factors.

From part (1) of Theorem 4.1 we have for $n \geq 1$,

$$
\begin{aligned}
& L_{0}(z)-\frac{R_{n}(\omega ; z)}{S_{n}(\omega ; z)}=O\left(z^{n}\right), \\
& L_{\infty}(z)-\frac{R_{n}(\omega ; z)}{S_{n}(\omega ; z)}= \begin{cases}O\left(\frac{1}{z^{n+1}}\right), & \text { when } \omega=0, \\
O\left(\frac{1}{z^{n-1}}\right), & \text { when } \omega=1, \\
O\left(\frac{1}{z^{n}}\right), & \text { otherwise. }\end{cases}
\end{aligned}
$$

Let the sequence of polynomials $\left\{\chi_{n}^{(1)}(\omega ; z)\right\}$ and $\left\{\chi_{n}^{(2)}(\omega ; z)\right\}$ be defined by

$$
\begin{aligned}
& \chi_{n}^{(1)}(\omega ; z)=R_{n}(\omega ; z) S_{n-1}(z)-R_{n-1}(z) S_{n}(\omega ; z), \\
& \chi_{n}^{(2)}(\omega ; z)=S_{n}^{\prime}(\omega ; z) S_{n-1}(z)-S_{n-1}^{\prime}(z) S_{n}(\omega ; z),
\end{aligned}
$$

From the recurrence relations (3.8) and (3.10),

$$
\begin{aligned}
\chi_{n}^{(1)}(\omega ; z)= & -2 \mu_{0} \delta_{1} \alpha_{2} \cdots \alpha_{n} z^{n-1}, \\
\chi_{n}^{(2)}(\omega ; z)= & \alpha_{n} \alpha_{n-1} z^{2} \chi_{n-2}^{(2)}(0 ; z) \\
& +(1-\omega)\left[S_{n-1}(z)\right]^{2}+\alpha_{n} \beta_{n-1}\left[S_{n-2}(z)\right]^{2},
\end{aligned}
$$

for $n \geq 2$, with $\chi_{1}^{(1)}(\omega ; z)=-2 \mu_{0} \delta_{1}, \alpha_{1} \chi_{0}^{(2)}(\omega ; z)=0$ and $\chi_{1}^{(2)}(\omega ; z)=1-\omega$. 
Theorem 4.3. Let $\delta_{n}=(-1)^{n} S_{n}(0)$ for $n \geq 1$, where $S_{n}$ are the Szegö polynomials associated with the sq-definite moment functional $\mathcal{M}$. Then for $\omega \neq 1$, the zeros of $S_{n}(\omega ; z)$ are the eigenvalues of the lower Hessenberg matrix

$$
\mathbf{H}_{n}=\left[\begin{array}{cccccc}
\eta_{1} & \alpha_{2} & 0 & \cdots & 0 & 0 \\
\eta_{1} & \eta_{2} & \alpha_{3} & \cdots & 0 & 0 \\
\vdots & \vdots & \vdots & & \vdots & \vdots \\
\eta_{1} & \eta_{2} & \eta_{3} & \cdots & \alpha_{n-1} & 0 \\
\eta_{1} & \eta_{2} & \eta_{3} & \cdots & \eta_{n-1} & \alpha_{n} /(1-\omega) \\
\eta_{1} & \eta_{2} & \eta_{3} & \cdots & \eta_{n-1} & \eta_{n} /(1-\omega)
\end{array}\right]
$$

where $\eta_{1}=\delta_{1}$ and $\eta_{r}=\delta_{r} \bar{\delta}_{r-1}, \alpha_{r}=\delta_{r} \bar{\delta}_{r-1}-\delta_{r} / \delta_{r-1}, r=2, \ldots, n$.

Proof. The proof of this theorem follows from (3.8) and results established in 14.

\section{Polynomials With Real zeros}

Let $\mathcal{M}$ be a rsq-definite moment functional. That is, all of the moments $\mu_{s}$, $s=0, \pm 1, \pm 2, \ldots$, are real and the properties (3.1) and (3.2) hold.

Clearly, the associated Szegö polynomials $S_{n}$ are all real and, in particular, we can state the following theorem.

Theorem 5.1. Let $\delta_{n}=(-1)^{n} S_{n}(0)$ for $n \geq 1$, where $S_{n}$ are the Szegö polynomials associated with the rsq-definite moment functional $\mathcal{M}$. Then all three statements below are equivalent:

(1) $H_{n}^{(-n)}>0$ for $n \geq 1$;

(2) $\quad \delta_{n}>1$ for $n \geq 1$;

(3) All the zeros of the polynomials $S_{n}(\omega ;),. n \geq 1$, when $\omega<1$, are distinct and lie on the positive half of the real line.

Proof. $\mathcal{M}$ being a rsq-definite moment functional means the moments are real and $H_{n}^{(-n+1)}>0, n \geq 1$. Hence from (3.7), $H_{n}^{(-n)}>0, n \geq 1$ implies $\delta_{n}>1, n \geq 1$. On the other hand, if $\delta_{n}>1, n \geq 1$, then the determinant condition $H_{n}^{(-n)}>0$, $n \geq 1$ follows from (2.11). Therefore (1) and (2) are equivalent.

Now we prove the equivalence of (2) and (3). Suppose that all the zeros of $S_{n}\left(\omega ;\right.$.) (for all $n \geq 1$ ) are positive. Since the leading coefficient $1-\omega$ of $S_{n}(\omega ;$.) is positive, $\delta_{n}=(-1)^{n} S_{n}(\omega ; 0)>0, n \geq 1$. Combining this with the information that $\mathcal{M}$ is an rsq-definite moment functional (2) is established. On the other hand, if $\delta_{n}>1, n \geq 1$, then from (4.4) $\chi_{n}^{(2)}(\omega ; z)>0, n \geq 1$ for $\omega<1$ and for any real values of $z$. Using this with the additional conditions $(-1)^{n} S_{n}(0)>0, n \geq 1$, we easily establish that all the zeros of $S_{n}(\omega ;$.$) are positive and distinct (and, even$ more, interlace with the zeros of $\left.S_{n-1}\right)$. This proves the equivalence of (2) and (3). Thus, proving the theorem. 
The recurrence relation (3.8) that corresponds to the conditions of the above theorem has been studied, for example, in [13].

Remark. One can also establish in an analogous manner (or using the polynomials $\left.T_{n}(z)=(-1)^{n} S_{n}(-z), n \geq 1\right)$ the equivalence of the statements

(1) $\quad(-1)^{n} H_{n}^{(-n)}>0$ for $n \geq 1$;

(2) $\quad(-1)^{n} \delta_{n}>1$ for $n \geq 1$;

(3) All the zeros of the polynomials $S_{n}(\omega ;),. n \geq 1$, when $\omega<1$, are distinct and lie on the negative half of the real line.

In fact, when $(-1)^{n} \delta_{n}>1, n \geq 1$, the polynomials $S_{n}$ are the denominator polynomials of a positive T-fraction. Thus we can also use known results on the denominator polynomials of a positive T-fraction given in [8].

From now on we restrict our analysis to the case when $\delta_{n}>1$ for $n \geq 1$ and we refer to the associated moment functional as an rsq-definite moment functional on $(0, \infty)$.

The results given from here on can be easily extended to the case when $(-1)^{n} \delta_{n}>$ $1, n \geq 1$, that is, when the moment functional is an rsq-definite moment functional on $(-\infty, 0)$.

Corollary 5.2. Let $\left\{S_{n}\right\}$ be the sequence of Szegö polynomials associated with $\mathcal{M}$ which is an rsq-definite moment functional on $(0, \infty)$. Then for the zeros $z_{n, r}(\omega)$ of $S_{n}(\omega ;$.$) we have$

$$
\begin{aligned}
& 0<z_{n, 1}(\omega)<z_{n, 2}(\omega)<\ldots<z_{n, n-1}(\omega)<z_{n, n}(\omega) \text { for } \omega<1, \\
& z_{n, 1}(\omega)<0<z_{n, 2}(\omega)<\ldots<z_{n, n-1}(\omega)<z_{n, n}(\omega) \text { for } \omega>1 .
\end{aligned}
$$

Proof. All we need to verify is that when $\omega>1$, the zeros of $S_{n}(\omega ;$.$) are still$ real and distinct and that one of the zeros is of the opposite sign. We verify this using the monic polynomial $(1-\omega)^{-1} S_{n}\left(\omega ;\right.$.). Substituting $z=0$ and $z=z_{n, r}(0)$, $r=1,2, \ldots, n$, in the definition of $S_{n}(\omega ;$.$) , we get$

$$
\prod_{r=1}^{n} z_{n, r}(\omega)=(1-\omega)^{-1} \prod_{r=1}^{n} z_{n, r}(0)
$$

and

$$
(1-\omega)^{-1} S_{n}\left(\omega ; z_{n, r}(0)\right)=\frac{\omega}{\omega-1} z_{n, r}(0) S_{n-1}\left(z_{n, r}(0)\right), \quad r=1,2, \ldots, n .
$$

Since the zeros of $S_{n}(0 ;)=.S_{n}$ are positive, distinct and interlace with the zeros of $S_{n-1}$, we can conclude that the zeros of $S_{n}(\omega ;$.$) interlace with the zeros of S_{n}$, proving that they are real and distinct. Moreover, at most, one zero of $S_{n}(\omega ;$.) can have the negative sign, which certainly happens if $(1-\omega)$ is negative.

Now we can state the following results on the quadrature rules associated with the polynomials $S_{n}(\omega ;$.$) .$

Theorem 5.3. Let $\omega<1$ and let $S_{n}$ be the Szegö polynomials associated with $\mathcal{M}$ which is an rsq-definite moment functional on $(0, \infty)$. Then the following hold: 


$$
\frac{R_{n}(\omega ; z)}{S_{n}(\omega ; z)}=\sum_{r=1}^{n} \frac{z_{n, r}(\omega)+z}{z_{n, r}(\omega)-z} \lambda_{n, r}(\omega), \quad n \geq 1,
$$

where $\quad \lambda_{n, r}(\omega)=\frac{R_{n}\left(\omega ; z_{n, r}(\omega)\right)}{-2 z_{n, r}(\omega) S_{n}^{\prime}\left(\omega ; z_{n, r}(\omega)\right)}$.

(2) $\quad \lambda_{n, r}(\omega)>0,1 \leq r \leq n \quad$ and $\quad \sum_{r=1}^{n} \lambda_{n, r}(\omega)=\mu_{0}$.

(3) For any $n \geq 1$, if $l_{p, q}(z)=\sum_{j=p}^{q} a_{j} z^{j}$ is a Laurent polynomial such that $-n+1 \leq p \leq q \leq n-1$, then the quadrature rule holds:

$$
\mathcal{M}\left[l_{p, q}\right]=\sum_{r=1}^{n} \lambda_{n, r}(\omega) l_{p, q}\left(z_{n, r}(\omega)\right) .
$$

Proof. To prove part (1), first observe that all the the zeros of $S_{n}(\omega ;$.$) are distinct$ and lie within $(0, \infty)$. Hence, a partial decomposition of the given type verifies part (1) of the theorem.

Note that $\lambda_{n, r}(\omega)$ can be written as

$$
\lambda_{n, r}(\omega)=\frac{-\chi_{n}^{(1)}\left(\omega ; z_{n, r}(\omega)\right) / z_{n, r}(\omega)}{2 \chi_{n}^{(2)}\left(\omega ; z_{n, r}(\omega)\right)},
$$

where $\chi_{n}^{(1)}(\omega ;$.$) and \chi_{n}^{(2)}(\omega ;$.$) are as in (4.4). Since \delta_{n}>1$ for $n \geq 1$, one can easily verify that the numerator and denominator above are positive and hence part (2) of the theorem is established.

When $n=1$, part (3) of this theorem clearly follows from part (2). To obtain part (3) when $n \geq 2$, we note that $\tilde{l}_{p, q}(t)=t^{n-1} l_{p, q}(t) \in \mathbb{P}_{2 n-2}$. Hence, the interpolation on the zeros of $S_{n}(\omega ;$.$) gives,$

$$
\begin{aligned}
\tilde{l}_{p, q}(t)=\sum_{r=1}^{n} \frac{S_{n}(\omega ; t)}{\left(t-z_{n, r}(\omega)\right) S_{n}^{\prime}\left(\omega ; z_{n, r}(\omega)\right)} & \tilde{l}_{p, q}\left(z_{n, r}(\omega)\right) \\
& +\tilde{l}_{p, q}\left[z_{n, 1}(\omega), \ldots, z_{n, n}(\omega), t\right] S_{n}(\omega ; t) .
\end{aligned}
$$

Here, the divided difference $\tilde{l}_{p, q}\left[z_{n, 1}(\omega), \ldots, z_{n, n}(\omega), t\right] \in \mathbb{P}_{n-2}$ and therefore, $\mathcal{M}\left[t^{-n+1} \tilde{l}_{p, q}\left[z_{n, 1}(\omega), \ldots, z_{n, n}(\omega), t\right] S_{n}(\omega ; t)\right]=0$. This means,

$$
\mathcal{M}\left[l_{p, q}\right]=\mathcal{M}\left[t^{-n+1} \tilde{l}_{p, q}\right]=\sum_{r=1}^{n} \tilde{\lambda}_{n, r}(\omega)\left(z_{n, r}(\omega)\right)^{n-1} l_{p, q}\left(z_{n, r}(\omega)\right),
$$

where $\tilde{\lambda}_{n, r}(\omega)=\mathcal{M}\left[\frac{t^{-n+1} S_{n}(\omega ; t)}{\left(t-z_{n, r}(\omega)\right) S_{n}^{\prime}\left(\omega ; z_{n, r}(\omega)\right)}\right]$. Since, one can also establish that

$$
R_{n}(\omega ; z)+2 z^{n} \mathcal{M}\left[\frac{t^{-n+1} S_{n}(\omega ; t)}{t-z}\right]=S_{n}(\omega ; z) \mathcal{M}\left[\frac{t+z}{t-z}\right],
$$

we obtain the required result of the theorem.

Moreover, using the results given in Theorem 4.2 and Theorem 5.3 the following results can also be easily verified. If $\omega<1$ and $\nu=\frac{1-\omega-\left|\delta_{n}\right|^{2}}{1-\omega}$, then for $n \geq 1$,

$$
z_{n, r}(\omega)=1 / z_{n, n-r+1}(\nu) \quad \text { and } \quad \lambda_{n, r}(\omega)=\lambda_{n, n-r+1}(\nu), \quad r=1,2, \ldots, n .
$$


The results in Theorem 5.3 permits one to define the step function $\psi_{n}(\omega ;$.$) by$

$$
\psi_{n}(\omega ; t)= \begin{cases}0, & 0<t \leq z_{n, 1}(\omega) \\ \sum_{r=1}^{s} \lambda_{n, r}(\omega), & z_{n, s}(\omega)<t \leq z_{n, s+1}(\omega), \\ \mu_{0}, & z_{n, n}(\omega)<t<\infty\end{cases}
$$

Then from part (1) of Theorem 5.3 we get

$$
\frac{R_{n}(\omega ; z)}{S_{n}(\omega ; z)}=\int_{0}^{\infty} \frac{t+z}{t-z} d \psi_{n}(\omega ; t), \quad n \geq 1
$$

and hence from (4.3), for $n \geq 1$,

$$
\mu_{s}=\int_{0}^{\infty} t^{s} d \psi_{n}(\omega ; t), \quad-n+1 \leq s \leq n-1
$$

Hence, using the Helly selection theorem there exists a subsequence $\left\{n_{k}\right\}$ such that $\left\{\psi_{n_{k}}(\omega)\right\}$ converges to a bounded non-decreasing function $\psi$ defined on the positive half of the real line. The function $\psi$ is such that it has infinitely many points of increase in $(0, \infty)$ and

$$
\lim _{k \rightarrow \infty} \frac{\left.R_{n_{k}} \omega ; z\right)}{S_{n_{k}}(\omega ; z)}=\lim _{k \rightarrow \infty} \int_{0}^{\infty} \frac{t+z}{t-z} d \psi_{n_{k}}(\omega ; t)=\int_{0}^{\infty} \frac{t+z}{t-z} d \psi(t),
$$

for $0<\arg (z)<2 \pi$. The convergence is also uniform for any compact subsets within $0<\arg (z)<2 \pi$.

The points of increase (support) of $\psi$ lie entirely on the positive half of the real line.

Moreover,

$$
\mu_{s}=\int_{0}^{\infty} t^{s} d \psi(t), \quad s=0, \pm 1, \pm 2, \ldots
$$

and, for all $n \geq 1$,

$$
\begin{aligned}
\int_{0}^{\infty} t^{-m} S_{n}(t) d \psi(t) & =\int_{0}^{\infty} t^{-n+m} S_{n}^{*}(t) d \psi(t) \\
& =\delta_{n, m} \frac{\Delta_{n}}{\Delta_{n-1}}, \quad 0 \leq m \leq n .
\end{aligned}
$$

The uniqueness of $\psi$ depends on the convergence of the continued fraction (2.4), where $\delta_{n}=S_{n}(0)$; see 8 ].

Assuming $\mathcal{M}$ to be an rsq-definite moment functional on $(0, \infty)$, we now consider the sequence of polynomials $\left\{S_{n}\left(\hat{\omega}_{n} ; z\right)\right\}$, where $\hat{\omega}_{n}=1-\delta_{n}$. The zeros of $S_{n}\left(\hat{\omega}_{n} ; z\right)$ are on the positive half of the real line. Using the results given in Theorem 4.2, we can say more about these zeros and also the quadrature weights $\lambda_{n, r}\left(\hat{\omega}_{n}\right)$ that follow from Theorem 5.3 .

$$
z_{n, r}\left(\hat{\omega}_{n}\right)=1 / z_{n, n-r+1}\left(\hat{\omega}_{n}\right), \quad \lambda_{n, r}\left(\hat{\omega}_{n}\right)=\lambda_{n, n-r+1}\left(\hat{\omega}_{n}\right), \quad r=1,2, \ldots, n,
$$

for $n \geq 1$. This means, the distribution given by the step function $\psi_{n}\left(\hat{\omega}_{n} ;\right.$.) satisfies

$$
d \psi_{n}\left(\hat{\omega}_{n} ; t\right)=-d \psi_{n}\left(\hat{\omega}_{n} ; 1 / t\right) \quad \text { for } \quad t \in(0, \infty) .
$$

Hence, by applying the Helly selection theorem we can state the following. 
Theorem 5.4. Let $\delta_{n}=(-1)^{n} S_{n}(0)$, where $S_{n}$ are the Szegö polynomials associated with $\mathcal{M}$ which is an rsq-definite moment functional on $(0, \infty)$. Then there exists a bonded non-decreasing function $\psi$, with all its points of increase on $(0, \infty)$, such that $d \psi(t)=-d \psi(1 / t)$.

Moreover,

$$
\mu_{s}=\int_{0}^{\infty} t^{s} d \psi(t) \quad \text { for } \quad s=0, \pm 1, \pm 2, \ldots
$$

and for $n \geq 1$,

$$
\int_{0}^{\infty} t^{-m} S_{n}(t) d \psi(t)=\int_{0}^{\infty} S_{m}^{*}(t) S_{n}(t) d \psi(t)=\delta_{n, m} \frac{\Delta_{n}}{\Delta_{n-1}}, \quad 0 \leq m \leq n .
$$

We now look at some properties of the sequences of polynomials $\left\{S_{n}\left(\hat{\omega}_{n} ;.\right)\right\}$ and $\left\{S_{n}\left(\check{\omega}_{n} ;.\right)\right\}$, where $\hat{\omega}_{n}=1-\delta_{n}$ as above and $\check{\omega}_{n}=1+\delta_{n}$, when $\mathcal{M}$ is an rsq-definite moment functional on $(0, \infty)$. From Theorem 4.2 and Corollary 5.2 , the only zero of $S_{n}\left(\check{\omega}_{n} ;.\right)$, which is negative, is equal to -1 .

In 4.2, letting $\sigma=\delta_{n} /\left|\delta_{n}\right|$ and $\sigma=-\delta_{n} /\left|\delta_{n}\right|$, we have for the monic polynomials $S_{n}\left(\hat{\omega}_{n} ; z\right) /\left|\delta_{n}\right|$ and $-S_{n}\left(\check{\omega}_{n} ; z\right) /\left|\delta_{n}\right|$,

$$
\frac{S_{n}\left(\hat{\omega}_{n} ; z\right)}{\left|\delta_{n}\right|}=\frac{S_{n}(z)+\tau_{n} S_{n}^{*}(z)}{1+\left|\delta_{n}\right|} \text { and } \quad \frac{S_{n}\left(\check{\omega}_{n} ; z\right)}{-\left|\delta_{n}\right|}=\frac{S_{n}(z)-\tau_{n} S_{n}^{*}(z)}{1-\left|\delta_{n}\right|},
$$

for $n \geq 1$, where $\tau_{n}=(-1)^{n} \delta_{n} /\left|\delta_{n}\right|$. Hence, from part 1 of Theorem 3.1] we obtain

$$
\begin{aligned}
\frac{S_{n+1}\left(\hat{\omega}_{n+1} ; z\right)}{\left|\delta_{n+1}\right|}= & \left(z-\frac{\left|\delta_{n}\right|}{\delta_{n}} \frac{\delta_{n+1}}{\left|\delta_{n+1}\right|}\right) \frac{S_{n}\left(\hat{\omega}_{n} ; z\right)}{\left|\delta_{n}\right|} \\
& -\frac{\left|\delta_{n}\right|}{\delta_{n}} \frac{\delta_{n+1}}{\left|\delta_{n+1}\right|}\left(\left|\delta_{n}\right|-1\right)\left(\left|\delta_{n-1}\right|+1\right) z \frac{S_{n-1}(z)+\tilde{\tau}_{n-1} S_{n-1}^{*}(z)}{1+\left|\delta_{n-1}\right|}, \\
\frac{S_{n+1}\left(\check{\omega}_{n+1} ; z\right)}{-\left|\delta_{n+1}\right|}= & \left(z-\frac{\left|\delta_{n}\right|}{\delta_{n}} \frac{\delta_{n+1}}{\left|\delta_{n+1}\right|}\right) \frac{S_{n}\left(\check{\omega}_{n} ; z\right)}{-\left|\delta_{n}\right|} \\
& -\frac{\left|\delta_{n}\right|}{\delta_{n}} \frac{\delta_{n+1}}{\left|\delta_{n+1}\right|}\left(\left|\delta_{n}\right|+1\right)\left(\left|\delta_{n-1}\right|-1\right) z \frac{S_{n-1}(z)-\tilde{\tau}_{n-1} S_{n-1}^{*}(z)}{1-\left|\delta_{n-1}\right|},
\end{aligned}
$$

where $\tilde{\tau}_{n-1}=(-1)^{n-1} \frac{\delta_{n}^{2}}{\left|\delta_{n}\right|^{2}} \frac{\left|\delta_{n+1}\right|}{\delta_{n+1}}$. Hence, these lead to three-term recurrence relations for $\frac{S_{n}\left(\hat{\omega}_{n} ; z\right)}{\left|\delta_{n}\right|}$ and $\frac{-S_{n}\left(\check{\omega}_{n} ; z\right)}{\left|\delta_{n}\right|}$, since $\tilde{\tau}_{n-1}=\tau_{n-1}$ when $\delta_{n}>1, n \geq 1$.

Theorem 5.5. Let $\delta_{n}=(-1)^{n} S_{n}(0)$, where $S_{n}$ are the Szegö polynomials associated with $\mathcal{M}$ which is an rsq-definite moment functional on $(0, \infty)$. Then

$$
\begin{aligned}
& \frac{S_{n+1}\left(\hat{\omega}_{n+1} ; z\right)}{\delta_{n+1}}=(z-1) \frac{S_{n}\left(\hat{\omega}_{n} ; z\right)}{\delta_{n}}-\left(\delta_{n}-1\right)\left(\delta_{n-1}+1\right) z \frac{S_{n-1}\left(\hat{\omega}_{n-1} ; z\right)}{\delta_{n-1}}, \\
& \frac{S_{n+1}\left(\check{\omega}_{n+1} ; z\right)}{-\delta_{n+1}}=(z-1) \frac{S_{n}\left(\check{\omega}_{n} ; z\right)}{-\delta_{n}}-\left(\delta_{n}+1\right)\left(\delta_{n-1}-1\right) z \frac{S_{n-1}\left(\check{\omega}_{n-1} ; z\right)}{-\delta_{n-1}},
\end{aligned}
$$

for $n \geq 1$, with $\frac{S_{1}\left(\hat{\omega}_{1} ; z\right)}{\delta_{1}}=z-1, \frac{S_{0}\left(\hat{\omega}_{0} ; z\right)}{\delta_{0}}=1, \frac{S_{1}\left(\check{\omega}_{1} ; z\right)}{-\delta_{1}}=z+1$ and $\frac{S_{0}\left(\check{\omega}_{0} ; z\right)}{-\delta_{0}}=0$. 


\section{Zeros AND CANONICAL SPECTRAL TRANSFORMATIONS}

Let $\mathcal{M}$ be an rsq-definite moment functional on $(0, \infty)$ defined as in (3.1), (3.2) and satisfying the conditions of Theorem 5.1. That is,

$$
\mu_{-n}=\mu_{n}, \quad(-1)^{n(n+1) / 2} \Delta_{n}=H_{n+1}^{(-n)}>0, \quad n=0,1,2, \ldots
$$

and

$$
H_{n}^{(-n)}>0, \quad n=1,2, \ldots .
$$

If $S_{n}$ denote the $n$-th Szegő polynomial associated with this moment functional, then all the zeros $z_{n, r}$ of $S_{n}$ are positive and we arrange these zeros as follows:

$$
0<z_{n, 1}<z_{n, 2}<\cdots<z_{n, n-1}<z_{n, n} .
$$

Now, let

$$
K_{n}(z, y)=\sum_{j=0}^{n} \frac{S_{j}(z) S_{j}(y)}{\kappa_{j}},
$$

where $\kappa_{n}=\Delta_{n} / \Delta_{n-1}$, the $n$-th reproducing kernel polynomial associated with $\mathcal{M}$. Then, the Christoffel-Darboux formula for the real Szegö polynomials is given by (see [15])

$$
K_{n}(z, y)=\frac{S_{n+1}^{*}(y) S_{n+1}^{*}(z)-S_{n+1}(y) S_{n+1}(z)}{\kappa_{n+1}(1-y z)} .
$$

In what follows, we denote by $z_{n, j}^{*}$ and $k_{n, j}$ the zeros of $S_{n}^{*}(z)$ and $K_{n}(\alpha, z)$, respectively. Interesting inequalities between zeros of these polynomials follow from the Theorem 3.1 and from the formula (6.1). Our results reads as follows.

Theorem 6.1. The zeros of $S_{n}$ and $S_{n}^{*}$ interlace. Precisely,

$$
0<z_{n, 1}^{*}<z_{n, 1}<z_{n-1,1}<\cdots<z_{n, n-1}^{*}<z_{n, n-1}<z_{n-1, n-1}<z_{n, n}^{*}<z_{n, n} .
$$

Proof. By Theorem 3.1, we have

$$
S_{n}(z)=(-1)^{n} \delta_{n} S_{n}^{*}(z)-\left(\left|\delta_{n}\right|^{2}-1\right) z S_{n-1}(z), \quad n \geq 1 .
$$

Since the zeros of $S_{n}(z)$ and $S_{n-1}(z)$ interlace, we obtain from the above relation

$$
\operatorname{sign}\left((-1)^{n} S_{n}^{*}\left(z_{n, k}\right)\right)=\operatorname{sign}\left(S_{n-1}\left(z_{n, k}\right)\right)
$$

and

$$
\operatorname{sign}\left(S_{n}\left(z_{n-1, k}\right)\right)=\operatorname{sign}\left((-1)^{n} S_{n}^{*}\left(z_{n-1, k}\right)\right) .
$$

Hence, there exist $z_{n, k}^{*}, k=1, \ldots, n$, zeros of $S_{n}^{*}(z)$, such that

$$
0<z_{n, 1}^{*}<z_{n, 1}<z_{n-1,1}<\cdots<z_{n, n-1}^{*}<z_{n, n-1}<z_{n-1, n-1}<z_{n, n}^{*}<z_{n, n} .
$$

Thus proving the theorem.

Theorem 6.2. If $\alpha<0$, then the zeros of $S_{n}$ and $K_{n}(\alpha,$.$) satisfy the interlacing$ property

$$
0<z_{n, 1}^{*}<z_{n, 1}<k_{n-1,1}<\cdots<z_{n, n-1}^{*}<z_{n, n-1}<k_{n-1, n-1}<z_{n, n}^{*}<z_{n, n} .
$$


Proof. By 6.1)

$$
\kappa_{n}(1-\alpha z) K_{n-1}(z, \alpha)=S_{n}^{*}(\alpha) S_{n}^{*}(z)-S_{n}(\alpha) S_{n}(z) .
$$

Now, since $(-1)^{n(n+1) / 2} \Delta_{n}>0$ and $\kappa_{n}=\Delta_{n} / \Delta_{n-1}$, we have $\operatorname{sign}\left(\kappa_{n}\right)=(-1)^{n}$. We also have $\operatorname{sign}\left(S_{n}(\alpha)\right)=(-1)^{n}$ and $S_{n}^{*}(\alpha)>0$. On the other hand, by Theorem 6.1. $S_{n}(z)$ and $S_{n}^{*}(z)$ have interlacing zeros. Therefore, from the above relation

$$
(-1)^{n} \operatorname{sign}\left(K_{n-1}\left(z_{n, j}, \alpha\right)\right)=\operatorname{sign}\left(S_{n}^{*}\left(z_{n, j}\right)\right), \quad 1 \leq j \leq n,
$$

and

$$
\operatorname{sign}\left(K_{n-1}\left(z_{n, j}^{*}, \alpha\right)\right)=-\operatorname{sign}\left(S_{n}\left(z_{n, j}^{*}\right)\right), \quad 1 \leq j \leq n .
$$

Hence there exist zeros $k_{n-1, j}, j=1, \ldots, n-1$, of $K_{n-1}(z, \alpha)$ satisfying $0<z_{n, 1}^{*}<$ $z_{n, 1}<k_{n-1,1}<\cdots<z_{n, n-1}^{*}<z_{n, n-1}<k_{n-1, n-1}<z_{n, n}^{*}<z_{n, n}$.

The analogues on the unit circle to the canonical spectral transformations on the real line have been introduced by Marcellán and co-workers; see [3, 5, 6, 9, 10] and are known in the literature by Christoffel, Geronimus and Uvarov. Our goal in this section is to study the zeros of polynomials generated by these three perturbations for Szegö. For this purpose, we denote by

$$
(p(z), q(z))_{\mathcal{M}}:=\mathcal{M}[p(z) q(1 / z)]
$$

the inner product associated with $\mathcal{M}$; here and below, we follow the notation of [3, 10].

6.1. Christoffel spectral transformation. The Christoffel transformation corresponding to a modification of the moment functional $\mathcal{M}$ is defined by

$$
\left.(p(z), q(z))_{\mathcal{M}_{C}}=((z-\alpha) p(z),(z-\alpha) q(z)]\right)_{\mathcal{M},}, \quad p, q \in \mathbb{P} .
$$

Notice that in our case $\alpha<0$.

Let $\left\{C_{n}\right\}_{n \geq 0}$ the sequences of monic orthogonal polynomials with respect to $\mathcal{M}_{C}$. We shall prove the zeros of the polynomials $C_{n}(z)$ are all real. Moreover, we obtain some inequalities. In order to do these, we recall the connections formula obtained in 3 ]

$$
(z-\alpha) C_{n}(z)=S_{n+1}(z)-\frac{S_{n+1}(\alpha)}{K_{n}(\alpha, \alpha)} K_{n}(z, \alpha) .
$$

Theorem 6.3. Denote by $c_{n, 1}, \ldots, c_{n, n}$ the zeros of $C_{n}(z)$. Then,

$$
0<z_{n+1,1}<k_{n, 1}<c_{n, 1}<\cdots<z_{n+1, n}<k_{n, n}<c_{n, n}<z_{n+1, n+1} .
$$

Proof. Since $S_{n+1}(\alpha) / K_{n}(\alpha, \alpha)<0$, from Theorem6.2 and formula (6.2) we obtain

$$
\operatorname{sign}\left(C_{n}\left(z_{n+1, j}\right)\right)=\operatorname{sign}\left(K_{n}\left(z_{n+1, j}, \alpha\right)\right), \quad 1 \leq j \leq n+1
$$

and

$$
\operatorname{sign}\left(C_{n}\left(k_{n, j}\right)\right)=\operatorname{sign}\left(S_{n+1}\left(k_{n, j}, \alpha\right)\right), \quad 1 \leq j \leq n .
$$

Hence there exist zeros $c_{n, j}, j=1, \ldots, n$, of $C_{n}(z)$ such that $0<z_{n+1,1}<k_{n, 1}<$ $c_{n, 1}<\cdots<z_{n+1, n}<k_{n, n}<c_{n, n}<z_{n+1, n+1}$. Thus, proving the theorem. 
6.2. Uvarov spectral transformation. We denoted by $\left\{U_{n}\right\}_{n \geq 0}$ the sequence of monic orthogonal polynomials associated with the Uvarov transformation

$$
(p(z), q(z))_{\mathcal{M}_{U}}=(p(z), q(z))_{\mathcal{M}}+m p(\alpha) q(\alpha), \quad \alpha= \pm 1 .
$$

The relation between the families of orthogonal polynomials was obtained in 3 . and it is given by

$$
U_{n+1}(z)=S_{n+1}(z)-\frac{m S_{n+1}(\alpha)}{1+m K_{n}(\alpha, \alpha)} K_{n}(z, \alpha) .
$$

Theorem 6.4. Denote by $u_{n, 1}(m), \ldots, u_{n, n}(m)$ the zeros of $U_{n}(z)$. Then, the followings statements hold:

(A) For every even $n$ we have

(i) $0<z_{n+1,1}<u_{n+1,1}(m)<k_{n, 1}<\cdots<z_{n+1, n}<u_{n+1, n}(m)<k_{n, n}<$ $z_{n+1, n+1}<u_{n+1, n+1}(m)$ if $\alpha=-1$ and $-1 / K_{n}(\alpha, \alpha)<m<0$. Moreover, all the zeros $u_{n+1, j}(m)$ decrease when $m$ increases, for $m \in\left(-1 / K_{n}(\alpha, \alpha), 0\right)$;

(ii) $u_{n+1,1}(m)<z_{n+1,1}<k_{n, 1}<\cdots<u_{n+1, n}(m)<z_{n+1, n}<k_{n, n}<$ $u_{n+1, n+1}(m)<z_{n+1, n+1}$ if $\alpha=-1$ and $m \in\left(-\infty,-1 / K_{n}(\alpha, \alpha)\right) \cup(0,+\infty)$. Moreover, all the zeros $u_{n+1, j}(m)$ are increasing functions of $m$, for $m<$ $-1 / K_{n}(\alpha, \alpha)$ or $m>0$, and

$$
\lim _{m \rightarrow \infty} u_{n+1,1}(m)=\alpha, \quad \lim _{m \rightarrow \infty} u_{n+1, j+1}(m)=c_{n, j}, \quad j=1, \ldots, n .
$$

(B) For every odd $n$ we have

(i) $0<z_{n+1,1}<u_{n+1,1}(m)<k_{n, 1}<\cdots<z_{n+1, n}<u_{n+1, n}(m)<k_{n, n}<$ $z_{n+1, n+1}<u_{n+1, n+1}(m)$ if $\alpha=-1$ and $0<m<-1 / K_{n}(\alpha, \alpha)$. Moreover, all the zeros $u_{n+1, j}(m)$ increase when $m$ increases, for $m \in\left(0,-1 / K_{n}(\alpha, \alpha)\right)$;

(ii) $u_{n+1,1}(m)<z_{n+1,1}<k_{n, 1}<\cdots<u_{n+1, n}(m)<z_{n+1, n}<k_{n, n}<$ $u_{n+1, n+1}(m)<z_{n+1, n+1}$ if $\alpha=-1$ and $m \in(-\infty, 0) \cup\left(-1 / K_{n}(\alpha, \alpha),+\infty\right)$. Moreover, all the zeros $u_{n+1, j}(m)$ are decreasing functions of $m$, for $m<0$ or $m>-1 / K_{n}(\alpha, \alpha)$, and

$$
\lim _{m \rightarrow \infty} u_{n+1,1}(m)=\alpha, \quad \lim _{m \rightarrow \infty} u_{n+1, j+1}(m)=c_{n, j}, \quad j=1, \ldots, n .
$$

Proof. The idea behind the proofs of these statements are similar, we only provide the proof of statement $(i)$ and $(i i)$ of $(A)$, which is much easier to follow. Suppose $\alpha=-1$. Note that $\operatorname{sign}\left(S_{n}(\alpha)\right)=(-1)^{n}$ and $\operatorname{sign}\left(K_{n}(\alpha, \alpha)\right)=(-1)^{n}$. Then, for every even $n$,

$$
\frac{m S_{n+1}(\alpha)}{1+m K_{n}(\alpha, \alpha)}>0
$$

for $-1 / K_{n}(\alpha, \alpha)<m<0$, and

$$
\frac{m S_{n+1}(\alpha)}{1+m K_{n}(\alpha, \alpha)}<0
$$

for $m<-1 / K_{n}(\alpha, \alpha)$ or $m>0$. Therefore, for $1 \leq j \leq n+1$,

$$
\operatorname{sign}\left(U_{n+1}\left(z_{n+1, j}\right)\right)=-\operatorname{sign}\left(K_{n}\left(z_{n+1, j}, \alpha\right)\right) \quad \text { if } \quad-1 / K_{n}(\alpha, \alpha)<m<0
$$

and

$$
\operatorname{sign}\left(U_{n+1}\left(z_{n+1, j}\right)\right)=\operatorname{sign}\left(K_{n}\left(z_{n+1, j}, \alpha\right)\right) \quad \text { if } m<-1 / K_{n}(\alpha, \alpha) \text { or } m>0 \text {. }
$$

Thus, the interlacing property $(i)$ and $(i i)$ of $(A)$ follows. For the monotonicity and the limit relations of the statements we apply Theorem 3.9 in 12 (see also Lemmas 1 and 2 of [4]). 
6.3. Geronimus spectral transformation. Let $\left\{g_{n}\right\}_{n \geq 0}$ be the sequence of orthonormal polynomials associated with the Geronimus transformation (see [1])

$$
(p(z), q(z))_{\mathcal{M}_{G}}=\left((z-\alpha)^{-1} p(z),(z-\alpha)^{-1} q(z)\right)_{\mathcal{M}}, \quad|\alpha|>1 .
$$

The sequence $\left\{G_{n}\right\}_{n \geq 0}$ given by $G_{n}=\left(\mathbf{k}_{n}\right)^{\frac{1}{2}} g_{n}$ is said to be the sequence of monic orthogonal polynomials associated with $\mathcal{M}_{G}$. These polynomials can be represented as

$$
G_{n+1}(z)=\left(z-\alpha \frac{\varepsilon_{n}(\alpha)}{\varepsilon_{n-1}(\alpha)}\right) S_{n}(z)+\frac{q_{n}(\alpha) q_{n}\left(\alpha^{-1}\right)}{\varepsilon_{n-1}(\alpha) \alpha^{n+1}} S_{n}^{*}(z), \quad n \geq 1,
$$

where

$$
\frac{\varepsilon_{n}(\alpha)}{\varepsilon_{n-1}(\alpha)}=\frac{\mathbf{k}_{n+1}}{k_{n}}, \quad n \geq 1
$$

and $\left\{q_{n}\right\}_{n \geq 0}$ are the so-called functions of the second kind associated with $\mathcal{M}_{G}$.

Theorem 6.5. The zeros of $G_{n}(z)$ are real for every fixed $\alpha$, with $|\alpha|>1$.

Proof. Without loss of generality, we suppose $\alpha<-1$. Then $\alpha \frac{\varepsilon_{n}(\alpha)}{\varepsilon_{n-1}(\alpha)}<z_{n, 1}<$ $\cdots<z_{n, n}$ which imply, by (6.4), that the polynomial $G_{n+1}(z)$ change sign $n+1$ times.

\section{REFERENCES}

1. A. K. Common and J. H. McCabe, The symmetric strong moment problem, J. Comput. Appl. Math., 67 (1996), 327-341. MR.1390189 (97e:30003)

2. C.F. Bracciali, J. H. McCabe and A. Sri Ranga, On a symmetric in strong distributions, J. Comput. Appl. Math., 105 (1999), 187-198. MR1690586 (2000e:30074)

3. L. Daruis, J. Hernández, and F. Marcellán, Spectral transformations for Hermitian Toeplitz matrices, J. Comput. Appl. Math., 202 (2007), 155-176. MR2319946 (2008c:42025)

4. D.K. Dimitrov, M.V. Mello, and F.R. Rafaeli, Monotonicity of zeros of Jacobi-Sobolev type orthogonal polynomials, Appl. Numer. Math., 60 (2010), 263-276. MR2602677(2011c:33016)

5. E. Godoy and F. Marcellán, An analogue of the Christoffel formula for polynomial modification of a measure on the unit circle, Boll. Un. Mat. Ital., 5-A (1991), 1-12. MR.1101005 (92c:42022)

6. E. Godoy and F. Marcellán, Orthogonal polynomials and rational modifications of measures, Canad. J. Math., 45 (1993), 930-943. MR1239908(95a:42031)

7. W.B. Jones, O. Njåstad and W.J. Thron, Moment theory, orthogonal polynomials, quadrature, and continued fractions associated with the unit circle, Bull. London Math. Soc., 21 (1989), 113-152. MR.976057(90e:42027)

8. W.B. Jones, W.J. Thron and H. Waadeland, A strong Stieltjes moment problem, Trans. Amer. Math. Soc., 261 (1980), 503-528. MR580900 (81j:30055)

9. X. Li and F. Marcellán, Representation of orthogonal polynomials for modified measures, Comm. Anal. Theory of Cont. Fract., 7 (1999), 9-22.

10. F. Marcellán and J. Hernández, Christoffel transforms and Hermitian linear functionals, Mediterr. J. Math., 2 (2005), 451-458. MR2192525 (2006h:42044)

11. F. Marcellán and J. Hernández, Geronimus spectral transforms and measures on the complex plane, J. Comput. Appl. Math., 219 (2008), 441-456. MR2441238 (2009k:42053)

12. F.R. Rafaeli, Zeros de Polinômios Ortogonais na Reta Real, Doctoral Thesis, UNICAMP, 2010 .

13. A. Sri Ranga, E.X.L. de Andrade and J.H. McCabe, Some consequences of symmetry in strong distributions, J. Math. Anal. Appl., 193 (1995), 158-168. MR.1338505 (97a:42022)

14. A.P. da Silva and A. Sri Ranga, Polynomials generated by a three term recurrence relation: bounds for complex zeros, Linear Algebra Appl., 397 (2005), 299-324. MR2116465 (2005m:33017) 
15. G. Szegő, Orthogonal polynomials, Amer. Math. Soc. Colloq. Publ., Vol. 23, Providence, RI, 1975. Fourth Edition. MR0372517 (51:8724)

16. L. Vinet and A. Zhedanov, Szegö polynomials on the real axis, Integr. Transforms and Spec. Functions, 8 (1999), 149-164. MR1730600(2000h:33010)

Departamento de Ciências de Computação e Estatística, IBilCE, Universidade Estadual Paulista - UNESP, Brazil

Current address: Departamento de Matemáticas, Escuela Politécnica Superior, Universidad Carlos III, Leganés-Madrid, Spain

E-mail address: kcastill@math.uc3m.es

Universidade Estadual de Mato Grosso do Sul - UEMS, Brazil

E-mail address: lamblem@uems.br

Departamento de Matemática, Estatística e Computação, FCT, Universidade EstaduAl PAulista - UNESP, Brazil

E-mail address: rafaeli@fct.unesp.br

Departamento de Ciências de Computação e Estatística, IBilCE, Universidade EsTAdual PAUlista - UNESP, Brazil

E-mail address: ranga@ibilce.unesp.br 